\title{
3 Research Square

\section{Regulation of Hippo/YAP signaling and esophageal squamous carcinoma progression by an E3 ubiquitin ligase PARK2}

\section{Xiaofeng Zhou}

Xinxiang Medical University SanQuan Medical College

\section{Yajie Li}

Xinxiang Medical University

\section{Weilong Wang}

Xinxiang Medical University

\section{Sujie Wang}

Xinxiang Medical University

Jinghan Hou

Xinxiang Medical University

Aijia Zhang

Xinxiang Medical University

\section{Benjie LV}

Xinxiang Medical University

\section{Can Gao}

Xinxiang Medical University

\section{Ziyi Yan}

Xinxiang Medical University

\section{Dan Pang}

Xinxiang Medical University

Kui Lu

Xinxiang Medical University

Nor Hazwani Ahmad

Universiti Sains Malaysia - Kampus Kesihatan

Jian Zhu

Xinxiang Medical University

Lidong Wang

Zhengzhou University

\section{Lichen Zhang}

Xinxiang Medical University

Ting Zhuang ( $\nabla 7090993 @ q q . c o m$ ) 
Xinxiang Medical University https://orcid.org/0000-0002-6308-1253

\section{Xiumin Li}

Xinxiang Medical University

\section{Research}

Keywords: PARK2, Hippo, YAP, ESCC, Ubiquitin

Posted Date: March 18th, 2020

DOI: https://doi.org/10.21203/rs.3.rs-17746/v1

License: (1) This work is licensed under a Creative Commons Attribution 4.0 International License. Read Full License 


\section{Abstract}

Background Esophageal squamous cell carcinoma (ESCC) is one of the most lethal malignancies in the world, while the overall five-year survival is less than $20 \%$. Recent genomic sequencing analysis indicated the over-activation of Hippo/YAP signaling might play important roles for the carcinogenic process and progression for ESCC patients. However, little is known about the molecular mechanisms that controls Hippo signaling activity in ESCC.

Methods YAP and PARK2 protein level were measured by western blot, while the Hippo classical target genes were measured by real-time PCR. WST1 assay were used to measure cell proliferation, the transwell and wound healing were used to measure the cell migration and invasion capacity. Protein stability and ubiquitin assay were used to detect the YAP protein ubiquitin and stability. The immuno-precipitation assays were used to detect the protein interactions. Immuno-staining was used to detect the protein localization of YAP and PARK2, while the ubiquitin-based immuno-precipitation assays were used to detect the specific ubiquitination manner of YAP.

Results Here, we identify a novel E3 ubiquitin ligase PARK2 as an inhibition factor for Hippo/YAP axis. We find that PARK2 depletion promotes ESCC progression both in vivo and in vitro through Hippo/YAP axis, while PARK2 overexpression suppresses ESCC tumor progression via Hippo signaling. Mechanistic study reveals that PARK2 could interact with YAP in the cytosol and promotes YAP K48-linked ubiquitination at K90 sites, which subsequently promotes YAP protein degradation in ESCC. Clinical sample of ESCC revealed that PARK2 is significantly decreased in ESCC, and relates to good prognosis in ESCC patients. Beside, PARK2 expression negatively correlates with tumor stage and YAP protein expression.

Conclusions: Our study identifies an interesting mechanism of Hippo pathway regulation, by which PARK2 modulates ESCC cancer progression, and implies PARK2 could be a novel marker for therapeutics and diagnostics in human ESCC.

\section{Background}

Esophageal cancer is the eighth malignancy in cancer incidence and mortality worldwide [1]. Among all the esophageal cancer patients, about $60 \%$ of them are diagnosed in China. According to the cancer statistics, over 300,000 new esophageal cancer cases happen in China [2]. Esophageal cancer in China exhibits a different pathological pattern compared with western countries, while esophageal squamous cell carcinoma (ESCC) is the major subtype. Besides, even with a high incidence of ESCC in China, there are dramatic high district variations in China mainland. Some districts, such as northern part of Henan province, have higher ESCC incidence [3]. Besides the environmental and living habit related factors, including tobacco and alcohol consumption, recent studies via genomic sequencing showed that the genetic alternations, such as gene mutations and amplifications, are pervasive in human ESCC samples and play important roles in carcinogenesis [4]. Interestingly, the genomic sequencing data showed that the deficiency of inhibitory factors of Hippo/YAP pathway, such as AJUBA and FATs mutations, or YAP 
gene amplification accounted for $48 \%$ of ESCC samples, indicating that dys-regulation of Hippo signaling could play critical role in ESCC progression [5]. However, the insights of the molecular mechanisms that control Hippo signaling activity and YAP/TEAD turnover are of utmost importance for ESCC diagnostics and therapeutics.

The Hippo signaling was initially identified from Drosophila [6]. Further studies showed that Hippo signaling is an evolutionarily conserved pathway, which modulates tissue growth and organ size in a range of species. The core of Hippo signaling is composed of a kinase cascade: the upstream phosphorylation kinase MST1/2 promotes LATS1/2 phosphorylation and activation, which subsequently phosphorylates the pathway effector YAP/TAZ and promotes YAP/TAZ retention in the cytosol and degradation [7]. But, If YAP/TAZ are not phosphorylated, they will trans-locate into the nuclear, interact with transcriptional factors, such as TEAD and RUNX to regulate genes involved in cell growth, migration, survival and metabolism [8, 9]. Not surprisingly, dys-regulation of Hippo signaling has been implicated in many human cancers, including esophageal cancer [10]. Several components of Hippo signaling are found mutated in ESCC, including FATs, AJUBA, STK3, LATS1 and DCHS1 [5, 11]. Beside, YAP gene amplification and elevated expression are also observed in ESCC [12]. Based on these findings, we can observe several possible or confirmed mechanisms that lead to the inappropriate YAP/TEAD activation in ESCC, such as mutations of the inhibitory factors of Hippo signaling or elevated YAP expression [13]. However, as obvious candidates emerge components of ubiquitin-proteasome system, having been shown to safeguard Hippo signaling and modulating cancer progression.

PARK2 (Parkin) was firstly found as the Parkinson disease-related gene [14]. In such a neurodegenerative disease, the mutations of PARK2 gene were common in Juvenile Parkinson disease [15]. Further studies revealed that park2 deficiency causes mitochondrial disorder and oxidative stress in neurons $[16,17]$. Besides, PARK2 forms an interesting regulation loop with P53 signaling, in which P53 promotes PARK2 gene expression, while PARK2 in turn inhibits P53 signaling via promoting P53 protein degradation [18, 19]. PARK2 is found to diminished expression in several human cancers [20-23]. However, high up to $90 \%$ of P53 gene was mutated in ESCC, indicating PARK2 not likely effects via P53 signaling in ESCC. Thus, the mechanism that PARK2 contributes to tumor suppression and the regulation of PARK2 in ESCC are not clear in ESCC.

Here, we demonstrated PARK2 as an important inhibitor for Hippo/YAP signaling. PARK2 was decreased in ESCC human samples, correlated with good prognosis in ESCC patients and negatively related to YAP expression. PARK2 inhibited ESCC cancer progression via Hippo/YAP axis. PARK2 was found to associate with YAP in the cytosol and promoted YAP K48-linked ubiquitination and degradation. Hence, PARK2 functions to safeguard hippo signaling activity in ESCC, which could be a promising marker for ESCC cancer diagnostics and therapeutics.

\section{Materials And Methods}




\section{Cell culture}

Esophageal carcinoma cell lines (EC9706 and KYSE150) were cultured in RPMI 1640 Medium (Biological Industries) containing 10\% fetal bovine serum (Biological Industries), 1\% penicillin/streptomycin (Invitrogen). HEK-293 cells were cultured in DMEM Medium supplemented with 10\% FBS and 1\%penicillin/streptomycin. EC9706 and KYSE150 cells were authenticated by short tandem repeat profiling (STR). STR profiling of our KYSE150 cells was found to be $100 \%$ consistent with the STR data of the KYSE150 from China Infrastructure of Cell Line Resources (Supplementary figure 1). EC9706 cell STR profiling data was not accessible in public databases including ATCC. Cells were regularly tested for mycoplasma using Lookout Mycoplasma PCR detection kit (MP0035, Sigma) and only used when negative.

\section{Generation of knockout}

The PARK2 sgRNA was designed, synthesized, and cloned to the pX460 cloning vectors. Then, 100 million ESCC cells were electroporated with $4 \mu \mathrm{g}$ of pX460 plasmid containing sgRNA using the Nucleofector $^{\mathrm{TM}} 2 \mathrm{~b}$ Device (Lonza). After the electroporation, cell population was sorted by flow cytometry. Single cell can be obtained and then added into 96-well plates. Further 10 days' culture was allowed for the single cell clone expansion. The clones were collected for PCR amplification and sequencing to analyze the gene mutation in the PARK2 sgRNA recognizing site. The PARK2 sgRNA sequences or siRNA sequence were shown in supplementary table 1 . The PARK2 KO sequence was shown in supplementary Figure 2.

\section{RNA isolation and quantitative real-time PCR (qRT-PCR)}

Total RNA was extracted with RNeasy Plus Mini Kit (Qiagen, Valencia, CA) following the manufacturer's specifications. Reverse transcription was performed using the RevertAid First Strand cDNA Synthesis Kit(Thermo, Lithuania). qRT-PCR was carried out using GoTaq® qPCR Master Mix (Promega, USA) and 7500 Fast Real-Time PCR System (Applied Biosystems, Singapore). GAPDH was used as an internal control. The sequence of the primers for qPCR was listed in Supplementary Table 2.

\section{Western blot}

Standard western-blot assays were used to analyze protein expression in cells. The following antibodies were used for assays: anti-Flag-M2 (A8592, Sigma, 1:1000), anti-HA (2013819001, Roche, 1:1000) antiMyc (9E10, Santa Cruz, 1:1000), anti-GAPDH (0411, Santa Cruz, 1:1000), anti-PARK2 (Abcam,ab15954), anti-YAP (63.7, Santa Cruz, 1:1000), Protein signals were detected with an ECL kit (Millipore Co., Billerica, Massachusetts, USA)

\section{Immunofluorescence (IF) staining}

Cells on the coverslips were fixed with $4 \%$ paraformaldehyde and incubated with the primary antibody against PARK2 (Santa cruz,sc-32282), YAP (CST,14074) at $4{ }^{\circ} \mathrm{C}$ overnight. After washing with PBS, cells 
were then incubated with fluorescence-conjugated secondary antibody (Invitrogen, Carlsbad, CA), and subsequently counterstained with DAPI (Life Technology). Images were captured after staining with antifade DAPI solution using a confocal laser-scanning microscope (Leica TCS SP8 STED).

\section{Co-IP assays}

Co-IP assays were performed according to standard protocols. For the co-IP of Flag-PARK2 and Myc-YAP proteins, anti-Flag (A2220, Sigma) and anti-Myc ((9E10, Santa Cruz) agarose beads (30 $\mu$ l) were used to pull down Flag-PARK2 and Myc-YAP, respectively. Beads were washed with PBST three times, and bound protein was denatured with $2 \times$ SDS sample buffer. The supernatants were collected and proceded to SDS-PAGE western blot analysis.

\section{Wound healing and Transwell assays}

For the wound healing assay, cells were cultured in a 6-well plates until confluent and then wounded with a sterile tip. The cells were captured at the indicated time points after scratching. The distances between the two edges of the scratched wound were measured using Image $J$ software. The trans-well system (8 $\mu \mathrm{m}$ pore size, Corning) was employed for cell migration and invasion assays. For migration assays, cells in serum-free medium were seeded into the upper chambers. For invasion assays, the upper chambers were coated with matrigel (BD Biocoat, USA).After $24 \mathrm{~h}$, cells that had migrated through to the bottom of the insert membrane were fixed, stained with crystal violet and counted under $\times 20$ objective lens. The experiments were repeated thrice.

\section{In vivo ubiquitination assays}

For in vivo ubiquitination assays, cells were transfected with vectors, including expressing Myc-YAP, FlagPARK2 and HA-Ub, respectively, for $24 \mathrm{~h}$. Cells were then treated with MG132 $(10 \mu \mathrm{M})$ for $6 \mathrm{~h}$, and the levels of Myc-YAP ubiquitination was determined by IP with an anti-Myc antibody followed by westernblot assays with an anti-HA antibody (2013819001, Roche,1:1000)

\section{In vivo tumorigenesis and metastasis assay}

For in vivo tumorigenic experiment, EC9706 cells $\left(4 \times 10^{6}\right)$ were injected into the right dorsal flank of 4week-old female BALB/c nude mice. Tumor formation in nude mice was monitored over a 4-week period. The tumor volume was calculated by the formula: tumor volume $=0.5 \times$ length $\times$ width $^{2}$.For in vivo metastasis assays, each experimental group consisted of 54 -week-old female BALB/c nude mice. Briefly, $2 \times 10^{6}$ cells were injected intravenously through the tail vein into mouse. The mice were killed 8 weeks after injection. Tumor nodules formed on the lung surfaces were macroscopically determined and counted. The lungs were excised and embedded in paraffin. Further, the tissue sections were stained with H\&E to visualize the structure.

\section{Luciferase reporter assays}


For TEAD luciferase activity assays, cells with ectopic HA-PARK2 expression and their control cells were transfected with the TEAD luciferase reporter vector for $24 \mathrm{~h}$. Cells were then harvested for assays. Luciferase reporter assays were performed using the dual luciferase assay kit (Promega). The pRL-null vector expressing renilla luciferase (Promega) was used as an internal control to normalize the transfection efficiency.

\section{Cycloheximide assay}

Cycloheximide was added into culture medium with the final concentration of $100 \mu \mathrm{mol} / \mathrm{L}$. Cell lysis were collected at 1.5, 3 and $4.5 \mathrm{~h}$ after the treatment of cycloheximide.

\section{Tissue microarray (TMA) and immunohistochemistry (IHC)}

223 cases of ESCC were selected for the TMA construction. All of these tissue samples were obtained from the First Affiliated Hospital of Xinxiang Medical University. All patients signed informed consent. No patients recruited in the study received preoperative treatments. The ESCC samples used in this study were authorized by the Committees for Ethical Review of Research at Xinxiang medical University. IHC was performed according to a standard streptavidin-biotin-peroxidase complex method Signals in tumor cells were visually quantified using a scoring system from 0 to 9 . The scores were obtained by multiplying the intensity of signals with the percentage of positive cells (signal: $0=$ no signal, $1=$ weak signal, $2=$ intermediate signal, and $3=$ strong signal; percentage: $0=0 \%, 1 \leq 25 \%, 2=25-50 \%$, and $3 \geq$ $50 \%)$. Low and high expression were defined as scores of $<6$ and $\geq 6$, respectively.

\section{Cell proliferation assay}

Cell proliferation was assessed by EdU incorporation and flow cytometry. Proliferating cells were determined by using the 5-ethynyl-20-deoxyuridine (EdU) assay kit (Ribobio, Guangzhou, China). For quantification analysis of the images, each data point represents the positive fluorescence area calculated from a minimum of five randomly chosen fields from three individual experiments. EdU incorporation flow cytometry assay was carried out according to the manufacturer's instructions. The experiments were performed in triplicate.

\section{Statistical analysis}

No specific statistical tests were used to predetermine the sample size. Statistical analysis was performed using GraphPad Prism 7 software or SPSS version 23.0 (SPSS, Inc., IL). Data were expressed as mean \pm s.e.m. Differences between two independent groups were tested with Student's t-test. Kaplan -Meier analysis with log-rank test was applied for survival analysis. The relation between PARK2 expression and clinicopathological characteristics was analyzed by Pearson $\chi 2$ test. Univariate and multivariate Cox proportional hazard regression models were used to evaluate the survival hazard using Cox proportional hazard model with a forward stepwise procedure. Differences were considered to be statistically significant when $\mathrm{P}<0.05\left({ }^{\star} \mathrm{P}<0.01 ;{ }^{\star \star} \mathrm{P}<0.001\right)$. 


\section{Results}

\section{PARK2 functions as a tumor suppressor in ESCC}

We firstly analyzed the expression of esophageal cancer from TCGA database (https://tcgadata.nci.nih.gov/tcga/). The public available data indicated that PARK2 decreased eight folds in the esophageal cancer compared with normal esophageal tissue (Fig. 1A). In order to confirm this finding, we investigated ESCC samples together with adjacent esophageal tissues and observed that PARK2 expression is significantly decreased in ESCC tumors (Fig. 1B). Based on 223 ESCC patients sample analysis via IHC (immunohistochemistry), we found that low PARK2 expression correlated with late tumor stage $(P<0.001)$, poor differentiation $(P<0.04)$ (Fig. 1D), lymph node $(P<0.001)$ and distant metastasis $(P=0.0087)$ (Table 1). Besides, cox analysis showed that several risk factors related to poor prognosis in ESCC patients, including poor differentiation $(P=0.015)$, tumor invasion depth $(P=0.001)$, lymph node metastasis $(P<0.001)$, distant metastasis $(P<0.001)$ and low PARK2 expression $(P<0.001)$. KMPLOT survival analysis showed that low PARK2 expression significantly correlated with poor prognosis $(P<0.001)$ (Fig. 1C).

We further investigated the function of PARK2 in two ESCC cell lines (EC9706 and KYSE150). We generated stable clones of PARK2 knocking-out cell lines originated from EC9706 and KYSE150 cells (Fig. $1 \mathrm{E})$. We measured the migration and invasion capacity via trans-well assays with permeable filter and basement membrane respectively. The trans-well assays demonstrated that PARK2 KO cells increased the migration and invasion capacity in both EC9706 and KYSE150 cell models (Fig. 1F and 1G). The wound healing assays showed similar results that PARK2 KO cells showed increased wound closure speed compared with wild type cells in both EC9706 and KYSE150 cell models (Fig.1 $1 \mathrm{H}$ and 1I). The cell growth assay showed that PARK2 KO cells dramatically increased the number of EdU positive cells compared with wild type cells in both EC9706 and KYSE150 cells (Fig. 1J and 1K). The in vivo tumor growth assay showed that PARK2 KO cells increased growth speed in EC9706 cell models (Fig. 1L). Besides, we generated the PARK2 overexpression cell lines via lenti-virus infection. PARK2 overexpression inhibited cell migration and invasion in EC9706 cells (Fig. 1M). The wound-healing assay showed that PARK2 overexpression inhibited the wound-healing speed (Fig.1N). The clone formation assay showed that PARK2 overexpression inhibited the clone formation capacity in EC9706 cells (Fig. 10). The in vivo tumor growth assay showed that PARK2 overexpression significantly inhibited tumor growth in xenograft mice models (Fig. 1P).

\section{PARK2 inhibits ESCC progression via Hippo/YAP axis}

In order to investigate the potential mechanism, we carried out RNA sequencing analysis in PARK2 WT and $\mathrm{KO}$ cells. The signaling pathway analysis showed that PARK2 KO activated several pathways, such as Hippo signaling and IL-6 pathway, while inhibited several tumor suppressor pathways, including AMPK signaling and P53 signaling (Fig. 2A). Figure 2B showed that a group of Hippo signaling target genes 
were increased in PARK2 KO cells, such as CTGF and CYR61 (Fig.2B). The immuno-blotting analysis showed that PARK2 KO increased YAP protein level in EC9706 and KYSE150 cells (Fig. 2C), while the classical Hippo/YAP target genes were increased by PARK2 knocking-out in both EC9706 and KYSE150 cells, such as CTGF and CYR 61 (Fig. 2D). Consistently, PARK2 overexpression inhibited YAP protein level and Hippo target gene expression (Fig. 2E). The luciferase assay showed that PARK2 overexpression inhibited TEAD responsive element activity (Fig. 2F).

In order to investigate the logic link between the cancer phenotype and Hippo/YAP signaling in PARK2 function, we carried out several rescue experiments. In figure 2G, PARK2 knocking-out in EC9706 cells could promote cell migration and invasion, which effect could be at least partial rescued by further YAP knocking down. The in vivo metastatic assay showed that PARK2 KO could promote lung metastasis in mice, which effect could be rescued by YAP knocking down (Fig. 2H). The xenograft tumor assay showed that YAP knocking down could rescue the increased tumor growth speed by PARK2 knocking out (Fig. 2l).

\section{PARK2 expression reversely relates with YAP level in human samples and interacts with YAP protein in the cytosol}

We further analyzed the expression level of PARK2 and YAP in human ESCC samples. The immunohistochemistry results showed that PARK2 negatively correlated with YAP protein level $(P<0.001)$ (Fig. 3A and 3B). Beside, such trend in YAP and PARK2 expression was also observed in xenograft tumor samples (Fig. 3C). immuno-staining showed that PARK2 was localized mainly in the cytosol, while YAP was located both in the cytosol and nuclear (Fig. 3D). Immuno-precipitation assay showed that YAP could interact with PARK2 (Fig. 3E). Further cell fraction separation assay showed that YAP interacted with PARK2 in the cytosol (Fig. 3F). In order to investigate the interaction domain between the two proteins, we made the sub-clone variants of PARK2 and YAP (Fig. $3 \mathrm{G}$ and $3 \mathrm{H}$ ). The immuno-precipitation assay showed that the RING domain at the C-terminal of PARK2 was required for its interaction with YAP, while the WW domain of YAP was responsible to associate with PARK2 (Fig. $3 \mathrm{G}$ and $3 \mathrm{H}$ ).

\section{PARK2 modulates YAP stability via ubiquitination dependent manner}

Since PARK2 functions as an E3 ubiquitin ligase, it is most likely for PARK2 to modulate YAP through ubiquitin-based manner. We firstly investigated the PARK2 effect on YAP stability via cycloheximide, which indicated that PARK2 KO significantly prolonged the half-life of YAP in EC9706 cells (Fig. 4A). However, the proteasome inhibitor MG132 could diminish the inhibition effect of YAP protein by PARK2 over-expression, which indicated such regulation was proteasome dependent (Fig. 4B). We further investigated the effect of PARK2 on YAP protein ubiquitination. The ubiquitination-based IP showed that PARK2 could promote the overall ubiquitination level and K48-linked ubiquitination level of YAP, but did not change the K63-linked ubiquitination of YAP (Fig.4C-4E).

\section{PARK2 facilitates YAP protein ubiquitination at K90 site, which effect depends on PARK2 E3 ligase activity}


We further mutated the C431 sites of PARK2, which is required for PARK2 E3 ligase activity. The ubiquitination-based assay showed the PARK2 ${ }^{\mathrm{C} 431 \mathrm{~A}}$ mutant form could not inhibit YAP protein level or induce the poly-ubiquitination of YAP in ESCC cells (Fig. 5A-5B). Besides, the migration and invasion trans-well assay showed that PARK2 WT could inhibit the migration and invasion of EC9706 cells, while the PARK2 ${ }^{\text {C431A }}$ mutant form could not (Fig. 5C). The EdU-based flow-cytometry showed that PARK2 wild type form could inhibit ESCC cell proliferation, while the PARK2 ${ }^{\mathrm{C} 431 \mathrm{~A}}$ mutant form showed little effect on cell proliferation (Fig. 5D). We further investigated the exact ubiquitin ligation sites of YAP by PARK2. Since YAP protein has 13 lysine sites, we made 13 mutant variants of YAP. The ubiquitin-based IP showed that PARK2 promoted YAP polyubiquitination mainly at K90 site (Fig. 5E).

\section{Discussion}

Our current study reports a famous RING family E3 ligase PARK2 functions as an endogenous inhibitor for HIPPO/YAP axis in ESCC cancer. PARK2 is dramatically decreased in human ESCC samples, relates to good prognosis in ESCC patients and correlates with YAP protein negativity via IHC staining. PARK2 inhibits ESCC cancer cell progression both in vivo and in vitro. Mechanism study shows that PARK2 promotes YAP K48-linked poly-ubiquitination and proteasome-dependent degradation in ESCC cells (Fig. 6). Based on these data, we can propose that the modulation of PARK2 expression or PARK2 activity could a strategy to treat YAP-driven ESCC patients.

The conserved Hippo pathway controls tissue hemostasis and organ size in several species [24]. Since Hippo signaling is an inhibitory pathway, the dys-function of Hippo inhibitors, such as AJUBA and FATs mutation, or Hippo signaling effector YAP/TAZ overexpression, which causes Hippo/YAP axis overactivation, could play critical roles in carcinogenic process of several human cancers [5]. Interestingly, one genomic-based study showed that the mutation rate of Hippo signaling ranks NO. 8 among all cancerrelated pathways, which might indicate the genomic events of Hippo signaling abnormality are common in human cancer. In addition, one genomic sequencing study based on Chinese ESCC patient samples revealed that ESCC genomic abnormality could be clustered into five pathways, including cell cycle signaling, histone modification pathway, Hippo signaling, Notch signaling and PI3K pathway [18]. The genomic abnormity events of Hippo signaling were existed in almost half of ESCC patients, which implicated the important role of Hippo signaling in ESCC carcinogenic process and cancer progression. Several molecular mechanism studies showed that YAP depletion inhibited ESCC cell migration and invasion both in vivo and in vitro $[25,26]$. Since ESCC is such dependent on Hippo signaling, the Hippo pathway could an "Oncogenic addiction" pathway for ESCC. YAP/TAZ could be promising drug target for ESCC cancer therapeutics, while understanding the potential mechanism how YAP protein is regulated might provide novel insight into ESCC treatment.

It has been well established that Hippo signaling activity is mainly regulated via controlling the function of Hippo effectors-YAP/TAZ [10]. The function of YAP is mainly regulated through its trans-location between the cytosol and the nuclear. The phosphorylation by the Hippo pathway kinases cascade retains YAP protein in the cytosol and promotes YAP protein degradation, whereas the upstream pathways could 
compromise the YAP phosphorylation level, which permits YAP to enter the nuclear and activate Hippo target genes [6]. However, the recent studies showed that the ubiquitin-proteasome system is important safeguard to prevent over-activation of human cancers. For example, SCF ${ }^{\text {-TRCP }}$ complex is important for YAP protein polyubiquitination and degradation [27], while our recent study showed that SHARPIN-the LUBAC (linear ubiquitination assembly complex) component, could also facilitate YAP protein K48-linked poly-ubiquitination and degradation in ESCC cells [28]. Our current study revealed a novel layer of Hippo pathway regulator-PARK2, which associated with YAP at its C-terminal, promoted YAP poly-ubiquitination and degradation, which controlled the YAP protein half-life, turnover and also the duration of Hippo signaling output in ESCC. In consistent, PARK2 decreased its expression eight folds compared with normal esophageal tissue. Besides, PARK2 related to good prognosis, Well-differentiation of ESCC and YAP protein negativity in ESCC samples. Coupled with these data, we can assume that loss of PARK2, which subsequently lead to Hippo/YAP over-activation, could be a critical factor for ESCC carcinogenesis and progression.

PARK2 (also named Parkin) is composed of four functional domains, including UBL domain, RING0 (unique PARKIN domain), RING1, IBR domain (RING-in-between-RING domain) and RING2 domain [29]. PARK2 has been shown to function as an E3 ubiquitin ligase and controls several stress response pathway, such as mitochondrial quality control and autophagy [30,31]. One interesting finding in PARK2 field is that the mutation of PARK2 genes are related with hereditary early onset Parkinson disease (PD) [32]. However PARK2 was observed to decrease in several human cancers, including glioblastoma, ovary cancer, lung cancer and breast cancer [20, 21, 33, 34]. It is also intriguing that patients with Parkinson disease have higher risk of other cancers, such as melanoma, which might indicate the involvement of PARK2 deletion/mutation in the carcinogenesis process [35]. Several mice model studies showed that PARK2 KO mice are susceptible to hepatocellular carcinoma and colon cancer, suggesting that PARK2 could be important contributor for oncogenic process [20,36]. However, our previous studies indicated that PLCE1-a important risk factor for ESCC-linked to ESCC progression via snail signaling [37, 38]. Interestingly, our unbiased RNA sequencing data between PLCE WT and PLCE knockout cells revealed that PARK2 was important downstream target of PLCE1-snail axis [37]. Not surprisingly, further investigation showed PARK2 was a tumor suppressor in ESCC from clinical aspects, while the molecular mechanisms revealed a novel regulatory manner: PARK2 modulated Hippo pathway via promoted YAP protein ubiquitination and degradation in ESCC cells. Hence, PARK2 expression or mutation status could be an interesting prediction or prognosis marker for ESCC cancer patients.

\section{Conclusions}

In conclusion, we have validated PARK2 as a tumor suppressor for ESCC both in clinical sample and experimental studies. We demonstrated that PARK2 depletion was pervasive in ESCC and related to poor survival. PARK2 associated with YAP protein in the cytosol, promoted YAP ubiquitination and proteasome-dependent degradation in ESCC cells. Our studies revealed a novel function of PARK2 in 
Hippo signaling in multiple layers. As a novel modulator for Hippo signaling, modulation of PARK2 activity or gene expression level could be an appealing strategy to treat esophageal cancer.

\section{Abbreviations}

ESCC

esophageal squamous cell carcinoma

YAP

yes-associated protein

TEAD

TEA domain transcriptional factor

RING

really interesting new gene

LATS

large tumor suppressor kinase

PARK2

Parkinson disease protein 2

\section{Declarations}

Ethics Approval and Consent to participate: This study was reviewed and approved by the Ethical Board at Xinxiang Medical University. This usage of clinical samples was reviewed and approved by the Ethical Board at the First affiliated Hospital of Xinxiang University with written informed consents from all the patients.

\section{Consent for publication}

All authors read the manuscript and agree for publication

\section{Availability of supporting data}

The RNA sequence data are deposited in the Gene Expression Omnibus (GEO) database (Assessing number: GSE143947). The other data could be requested from the corresponding author.

\section{Competing Interests}

There is no competing interesting to declaim. 


\section{Funding}

The project was supported from the National Natural Science Foundation of China (U1704169, 81972330, Xiumin Li); The Project of Science and Technology Department of Henan Province (182102310126, Xiumin Li); the National Science Foundation for Young Scientists of China (No. 81702725, Ting Zhuang); the National Science Foundation for Young Scientists of China (No. 81601360, Lichen Zhang); the National Natural Science Foundation of China (81872032, U1804262هLidong Wang); the Joint Fund of the National Natural Science Foundation of China (No.U1604190, Jian Zhu).

\section{Authors' contributions}

Xiaofeng Zhou, Yajie Li and Weilong Wang performed most of the bench work. Lidong Wang, Lichen Zhang, Ting Zhuang and Xiumin Li supervised the process of the study and performed the manuscript writing. Jinghan Hou, Aijia Zhang, Benjie Lv and Can Gao performed the xenograft mice study and IHC data analysis. Ziyi Yan, Dan Pang, Kui Lu and Nor Hazwani Ahmad performed the RNA-sequence data analysis. Jian Zhu contributed to the manuscript writing. All authors read and approve the final manuscript.

\section{Acknowledgement}

We thank all the members of Laboratory of Molecular Oncology in Xinxiang University for sharing valuable material and research support. We thank The First Affiliated Hospital of Xinxiang Medical University for the valuable cancer sample support.

\section{Author information}

Xinxiang Key Laboratory for Molecular Therapy of Cancer, Xinxiang Medical University, Xinxiang 453003, Henan Province, P.R. China

Xiaofeng Zhou, Yajie Li, Weilong Wang, Sujie Wang, Jinghan Hou, Aijia Zhang, Benjie Lv, Can Gao, Ziyi Yan, Dan Pang, Kui Lu and Xiumin Li

State key laboratory of esophageal cancer prevention \& treatment, The First Affiliated Hospital of Zhengzhou University, Zhengzhou 450001, Henan Province, P.R. China 
School of Laboratory Medicine, Henan Collaborative Innovation Center of Molecular Diagnosis and Laboratory Medicine, Xinxiang Medical University, Xinxiang 453003, Henan Province, P.R. China Jian Zhu, Lichen Zhang and Ting Zhuang

Oncological and Radiological Sciences, Advanced Medical and Dental Institute, Universiti Sains Malaysia, Bertam, 13200 Kepala Batas, Penang, Malaysia

Nor Hazwani Ahmad

\section{References}

1. Chen $\mathrm{R}$ et al: [Analysis of incidence and mortality of esophageal cancer in China, 2015]. Zhonghua yu fang yi xue za zhi [Chinese journal of preventive medicine] 2019, 53(11):1094-1097.

2. Zeng $\mathrm{H}$ et al: Esophageal cancer statistics in China, 2011: Estimates based on 177 cancer registries. Thoracic cancer 2016, 7(2):232-237.

3. Wang $\mathrm{H}$ et al: Health-related quality of life among rural residents aged 45-69 years in Hua County, Henan Province, China: Results of ESECC Trial for esophageal cancer screening with endoscopy. Chinese journal of cancer research = Chung-kuo yen cheng yen chiu 2018, 30(2):240-253.

4. Qin HD et al: Genomic Characterization of Esophageal Squamous Cell Carcinoma Reveals Critical Genes Underlying Tumorigenesis and Poor Prognosis. American journal of human genetics 2016, 98(4):709-727.

5. Gao YB et al: Genetic landscape of esophageal squamous cell carcinoma. Nature genetics 2014, 46(10):1097-1102.

6. Meng Z, Moroishi T, Guan KL: Mechanisms of Hippo pathway regulation. Genes \& development 2016, 30(1):1-17.

7. Harvey KF, Zhang X, Thomas DM: The Hippo pathway and human cancer. Nature reviews Cancer 2013, 13(4):246-257.

8. Zhang $L$ et al: The TEAD/TEF family of transcription factor Scalloped mediates Hippo signaling in organ size control. Developmental cell 2008, 14(3):377-387.

9. Passaniti A, Brusgard JL, Qiao Y, Sudol M, Finch-Edmondson M: Roles of RUNX in Hippo Pathway Signaling. Advances in experimental medicine and biology 2017, 962:435-448. 
10. Han Y: Analysis of the role of the Hippo pathway in cancer. Journal of translational medicine 2019, 17(1):116.

11. Sawada G et al: Genomic Landscape of Esophageal Squamous Cell Carcinoma in a Japanese Population. Gastroenterology 2016, 150(5):1171-1182.

12. Zhao $\mathrm{J}$ et al: Effect of YAP1 silencing on esophageal cancer. OncoTargets and therapy 2016, 9:31373146.

13. Wang $L$ et al: Unbalanced YAP-SOX9 circuit drives stemness and malignant progression in esophageal squamous cell carcinoma. Oncogene 2019, 38(12):2042-2055.

14. Tomac AC, Hoffer BJ: Assignment of the mouse Park2 (PARKIN), the homologue to a new human Parkinson candidate gene, to the telomeric region of mouse 17A3.2-3.3, by in situ hybridization. Cytogenetics and cell genetics 2001, 95(1-2):120-121.

15. Sanyal J et al: PARK2 and PARK7 Gene Polymorphisms as Risk Factors Associated with Serum Element Concentrations and Clinical Symptoms of Parkinson's Disease. Cellular and molecular neurobiology 2019.

16. Rub C, Wilkening A, Voos W: Mitochondrial quality control by the Pink1/Parkin system. Cell and tissue research 2017, 367(1):111-123.

17. LaVoie MJ, Cortese GP, Ostaszewski BL, Schlossmacher MG: The effects of oxidative stress on parkin and other E3 ligases. Journal of neurochemistry 2007, 103(6):2354-2368.

18. Zhang $\mathrm{C}$ et al: Parkin, a p53 target gene, mediates the role of $\mathrm{p} 53$ in glucose metabolism and the Warburg effect. Proceedings of the National Academy of Sciences of the United States of America 2011, 108(39):16259-16264.

19. Checler F, Alves da Costa C: Interplay between parkin and p53 governs a physiological homeostasis that is disrupted in Parkinson's disease and cerebral cancer. Neuro-degenerative diseases 2014, 13(23):118-121.

20. Poulogiannis $\mathrm{G}$ et al: PARK2 deletions occur frequently in sporadic colorectal cancer and accelerate adenoma development in Apc mutant mice. Proceedings of the National Academy of Sciences of the United States of America 2010, 107(34):15145-15150.

21. Xu L, Lin DC, Yin D, Koeffler HP: An emerging role of PARK2 in cancer. Journal of molecular medicine 2014, 92(1):31-42.

22. Lee $S$ et al: Multiple-level validation identifies PARK2 in the development of lung cancer and chronic obstructive pulmonary disease. Oncotarget 2016, 7(28):44211-44223.

23. Gupta A, Anjomani-Virmouni S, Koundouros N, Poulogiannis G: PARK2 loss promotes cancer progression via redox-mediated inactivation of PTEN. Molecular \& cellular oncology 2017, 4(6):e1329692.

24. Maugeri-Sacca M, De Maria R: The Hippo pathway in normal development and cancer. Pharmacology \& therapeutics 2018, 186:60-72. 
25. Song $S$ et al: Hippo coactivator YAP1 upregulates SOX9 and endows esophageal cancer cells with stem-like properties. Cancer research 2014, 74(15):4170-4182.

26. Song $S$ et al: The Hippo Coactivator YAP1 Mediates EGFR Overexpression and Confers Chemoresistance in Esophageal Cancer. Clinical cancer research : an official journal of the American Association for Cancer Research 2015, 21(11):2580-2590.

27. Zhao B, Li L, Tumaneng K, Wang CY, Guan KL: A coordinated phosphorylation by Lats and CK1 regulates YAP stability through SCF(beta-TRCP). Genes \& development 2010, 24(1):72-85.

28. Zhang A et al: SHARPIN Inhibits Esophageal Squamous Cell Carcinoma Progression by Modulating Hippo Signaling. Neoplasia 2019, 22(2):76-85.

29. Chen $Y$ et al: The $C$-terminus of PARK2 is required for its self-interaction, solubility and role in the spindle assembly checkpoint. Biochimica et biophysica acta 2012, 1822(4):573-580.

30. Imaizumi $Y$ et al: Mitochondrial dysfunction associated with increased oxidative stress and alphasynuclein accumulation in PARK2 iPSC-derived neurons and postmortem brain tissue. Molecular brain 2012, 5:35.

31. Kuroda $Y$, Mitsui T, Kunishige M, Matsumoto T: Parkin affects mitochondrial function and apoptosis in neuronal and myogenic cells. Biochemical and biophysical research communications 2006, 348(3):787-793.

32. Bonifati $V$ et al: Autosomal recessive early onset parkinsonism is linked to three loci: PARK2, PARK6, and PARK7. Neurological sciences : official journal of the Italian Neurological Society and of the Italian Society of Clinical Neurophysiology 2002, 23 Suppl 2:S59-60.

33. Letessier A et al: Correlated break at PARK2/FRA6E and loss of AF-6/Afadin protein expression are associated with poor outcome in breast cancer. Oncogene 2007, 26(2):298-307.

34. Duan $\mathrm{H}$ et al: PARK2 Suppresses Proliferation and Tumorigenicity in Non-small Cell Lung Cancer. Frontiers in oncology 2019, 9:790.

35. Hu HH et al: PARKIN Inactivation Links Parkinson's Disease to Melanoma. Journal of the National Cancer Institute 2016, 108(3).

36. Fujiwara $\mathrm{M}$ et al: Parkin as a tumor suppressor gene for hepatocellular carcinoma. Oncogene 2008, 27(46):6002-6011.

37. Zhai S et al: PLCE1 Promotes Esophageal Cancer Cell Progression by Maintaining the Transcriptional Activity of Snail. Neoplasia 2017, 19(3):154-164.

38. Wang LD et al: Genome-wide association study of esophageal squamous cell carcinoma in Chinese subjects identifies susceptibility loci at PLCE1 and C20orf54. Nature genetics 2010, 42(9):759-763.

\section{Tables}

Due to technical limitations, the tables are only available as a download in the supplemental files section.

\section{Figures}



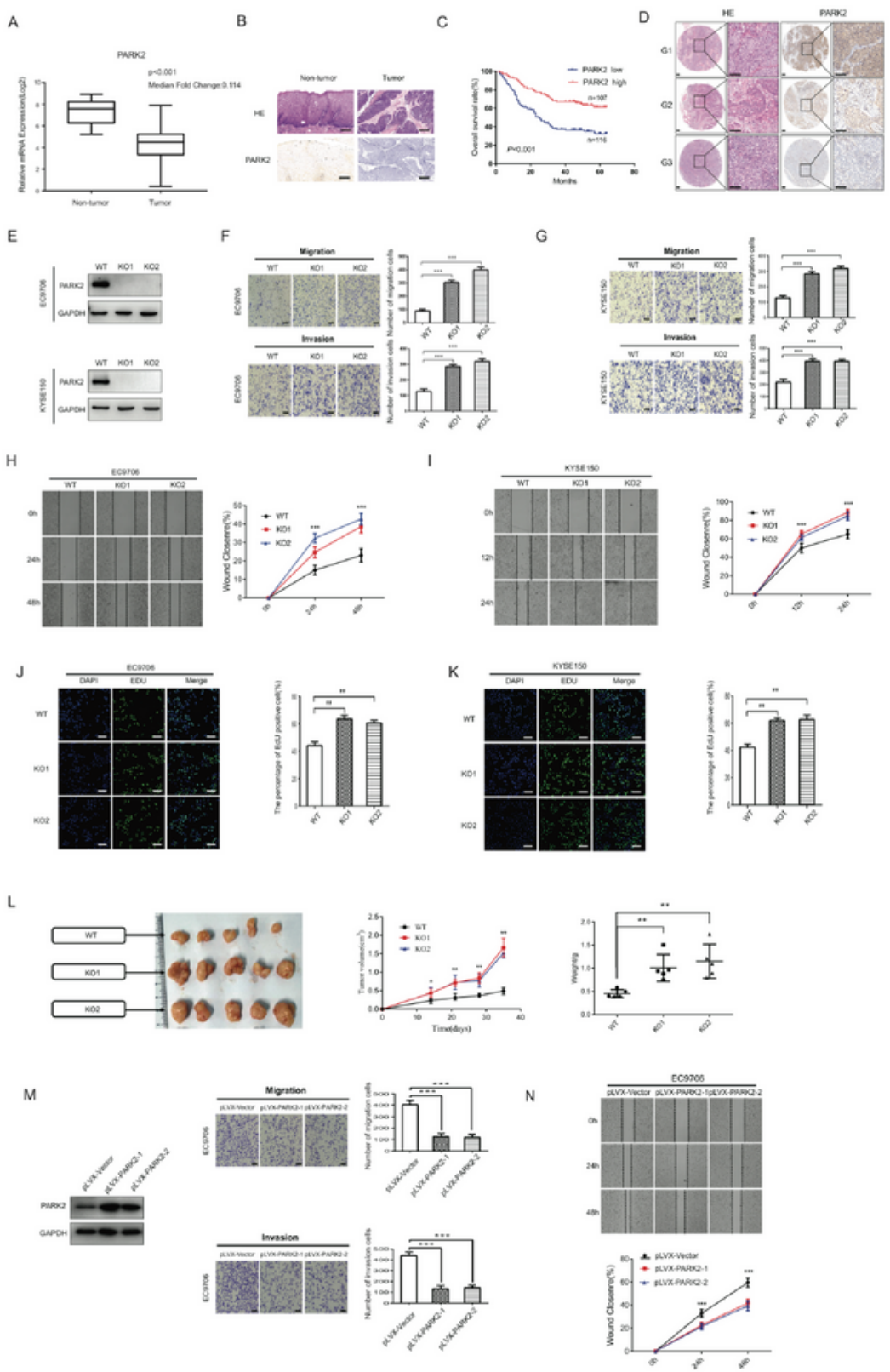

o
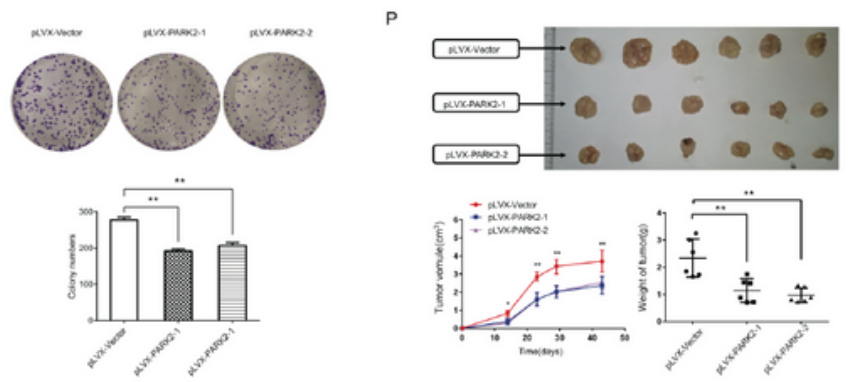

\section{Figure 1}

The implications of PARK2 in human ESCC samples and its effect on cancer-related phenotype in ESCC cell lines. A. PARK2 mRNA levels were significantly decreased in ESCC compared with matched adjacent non-tumor tissues. The data were obtained from TCGA database. B. PARK2 protein expression was significantly decreased in ESCC tissues compared with their adjacent non-tumor tissues as analyzed by IHC. C. Kaplan-Meier analysis revealed that low PARK2 expression was related with poorer overall 
survival of ESCC patients. P $<0.001$, log-rank test. D. H \& E staining was used to show different differentiation statuses of the ESCC (G1: high differentiation;G2: middle differentiation;G3:Iow differentiation). E. Immuno-blots showing CRISPR-mediated deletion of PARK2 in ESCC cell lines. F and G. PARK2 knockout promoted the migration and invasion in EC9706 cells( F) and KYSE150 cells (G) as determined by transwell assays. $\mathrm{H}$ and I. PARK2 knockout increased the wound-healing ability in EC9706 cells(H)and KYSE150 cells(I) compared with the wild type cells. J and K. EC9706 cells (J) and KYSE150 cells (K) were labeled with EdU. EdU-positive cells, green; cell nuclei, blue; scale bar 100 $\mu \mathrm{m}$. L. PARK2 knockout promoted the tumor growth of EC9706 cells in a xenograft model. The growth of xenografts was monitored over 5 weeks. Xenograft tumors were then dissected and their weights determined. $\mathrm{M}$ and $\mathrm{N}$. Representatives and summary of migration and invasion assay showing that overexpression of PARK2 inhibited cell migration and invasion in EC9706 cells. O. Colony formation ability decreased in PARK2 overexpression cells compared with control cells in EC9706 cells. P. PARK2 overexpression delayed the tumor growth of EC9706 cells in a xenograft model.

A

\begin{tabular}{|c|c|}
\hline Activated Pathway by PARK2 KO & Suppressed Pathway by PARK2 KO \\
\hline Hippo signaling & $\begin{array}{l}\text { Cell Cycle_G2_M DNA Damage Chockpoint } \\
\text { Requlation }\end{array}$ \\
\hline IL6 signaling & $\begin{array}{l}\text { Cell Cycle_G2_M DNA Damage Checkpoint } \\
\text { Regulation }\end{array}$ \\
\hline JAK-STAT signaling & EIF2 Signaling \\
\hline Neurotrophin_TRK signaling & TREM1 Signaling \\
\hline Prolactin Signaling & AMPK Signaling \\
\hline CDK5 signaling & p53 Signaling \\
\hline Endothelin-1 Signaling & CXCR4 Signaling \\
\hline Chemokine Signaling & NRF2-mediated Oxidative Stress Response \\
\hline TNFR2 Signaling & IL-3 Signaling \\
\hline HGF Signaling & PPAR Signaling \\
\hline
\end{tabular}

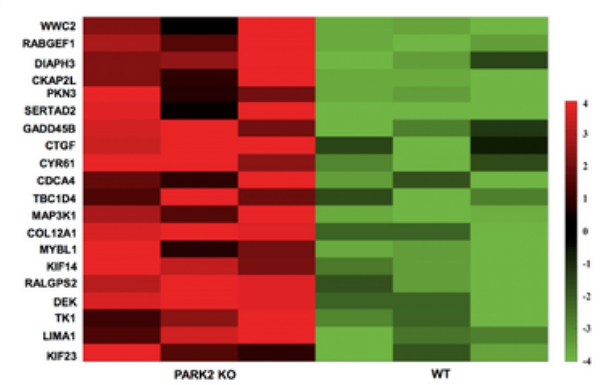

C

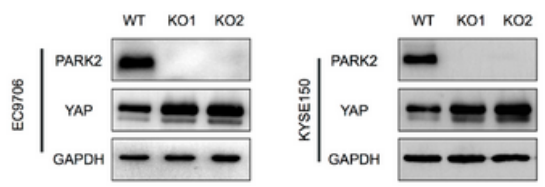

D
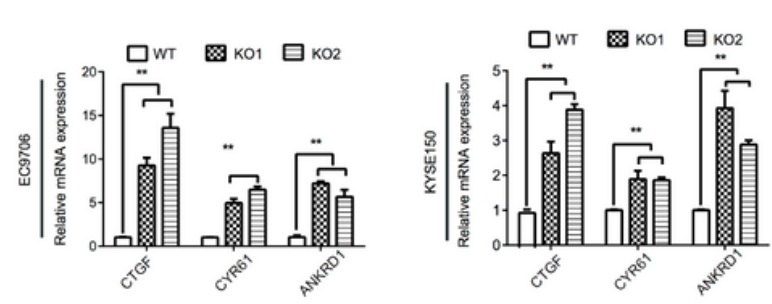

E
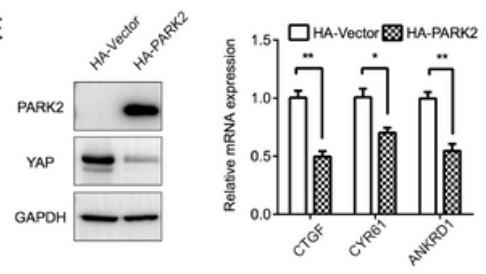

F

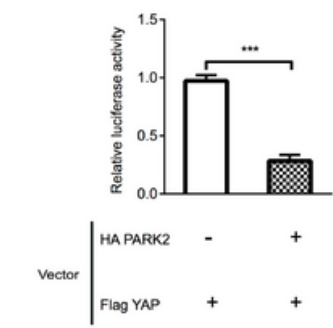

G

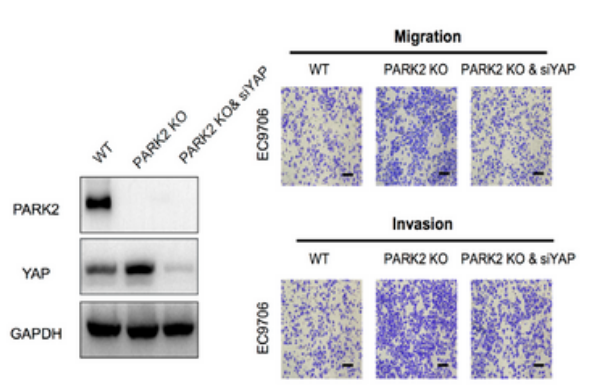

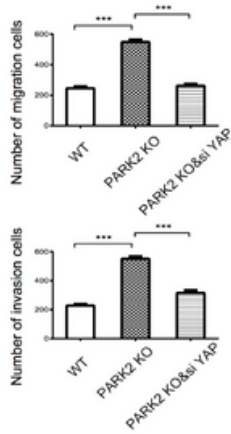

$\mathrm{H}$

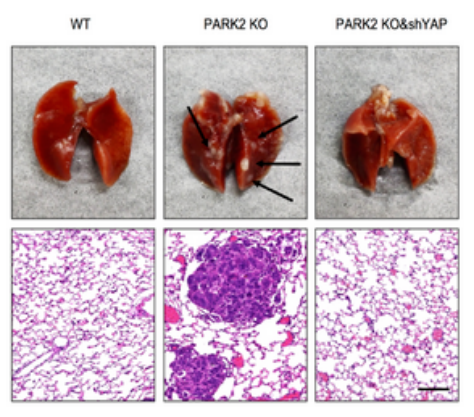

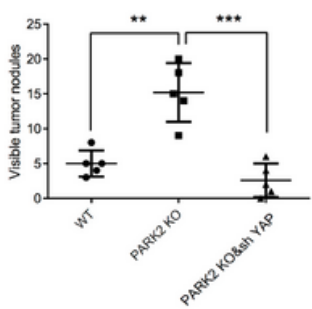

I
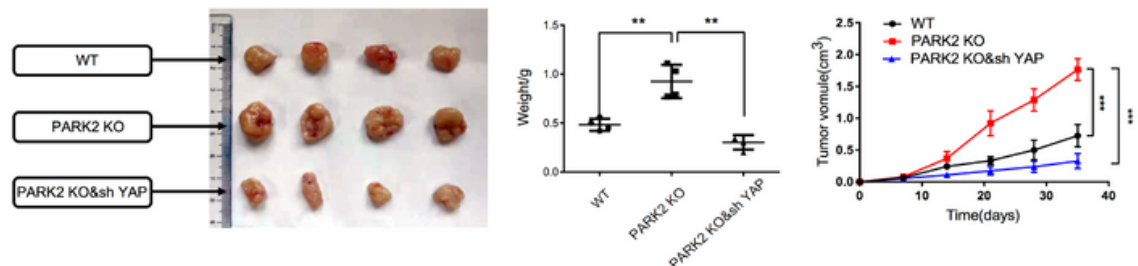


\section{Figure 2}

PARK2 inhibits ESCC progression in vivo and in vitro via Hippo signaling. A. KEGG pathway assay of differential mRNA transcripts in PARK2 KO clones identified by RNA-seq. B. Heat map of mRNA changes in WT and PARK2 KO single clones of EC9706 by bulk RNA-seq. C. Western blotting assays of PARK2 in WT and PARK2 KO single clones of EC9706 and KYSE150 cells. D. PARK2 KO increased mRNA expression of Hippo target genes in cells. E. HA-PARK2 expression reduced mRNA expression of Hippo target genes in EC9706 cells. F. HA-PARK2 expression inhibited TEAD luciferase reporter activities in EC9706 cells. G. Knockdown of YAP rescued the migration and invasion ability of cells with the PARK2 knockout. Scale bar; $100 \mu \mathrm{m}$. H. Knockdown of YAP rescued metastasis ability of cells with the PARK2 knockout in vivo. Black arrow indicates the pulmonary metastatic nodule. Scale bar, $100 \mu \mathrm{m}$. I. Knockdown of YAP rescued xenograft tumor growth of cells with the PARK2 knockout in vivo. ${ }^{\star \star} p<0.01$, ${ }^{\star \star \star} p<$ 0.001 . 
A

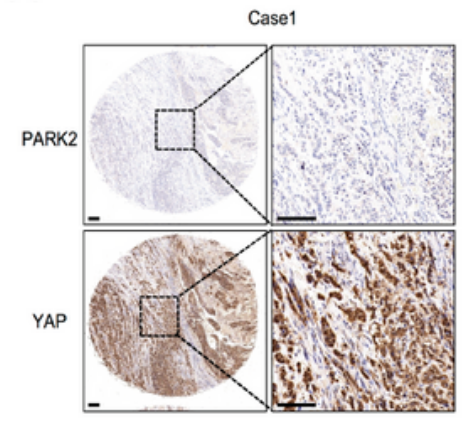

B

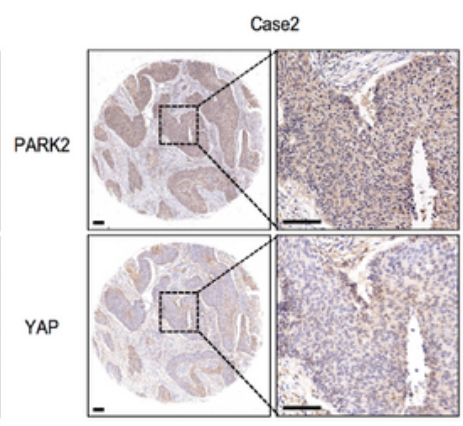

\begin{tabular}{cccc}
\hline \multirow{2}{*}{ PARK2 expression } & \multirow{2}{*}{ Cases } & \multicolumn{2}{c}{ YAP expression } \\
\cline { 3 - 4 } & & Low(\%) & High(\%) \\
\hline Low & 116 & $38(39.2 \%)$ & $78(60.8 \%)$ \\
High & 107 & $59(61.9 \%)$ & $48(38.1 \%)$ \\
\hline & & & $P<0.001, \chi^{2}$ test
\end{tabular}

C

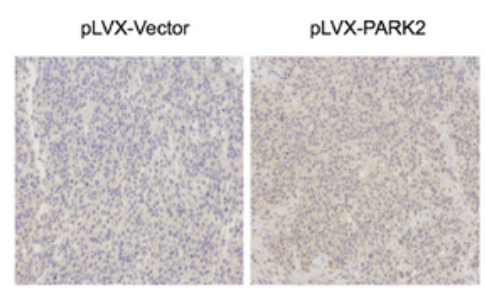

PARK2

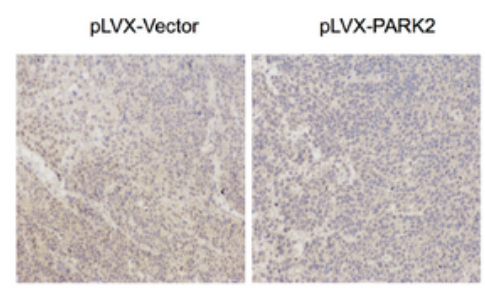

YAP

E

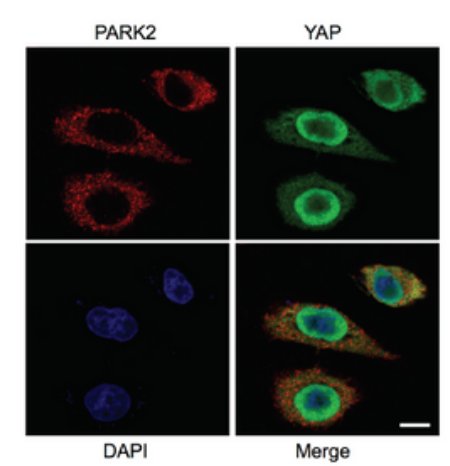

G

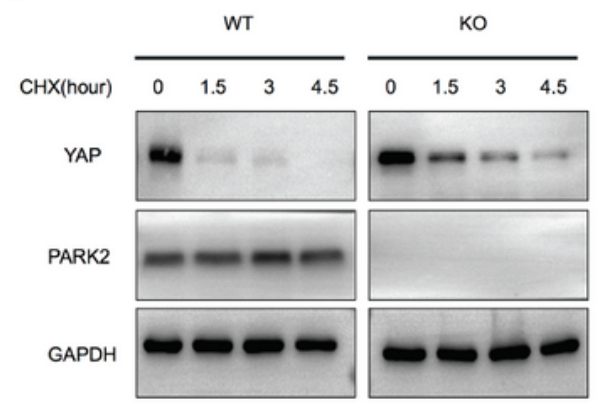

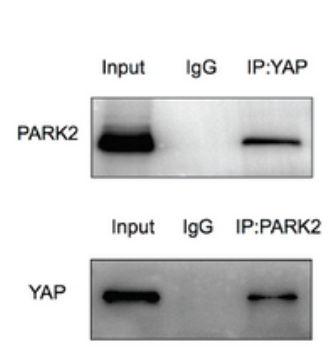

F

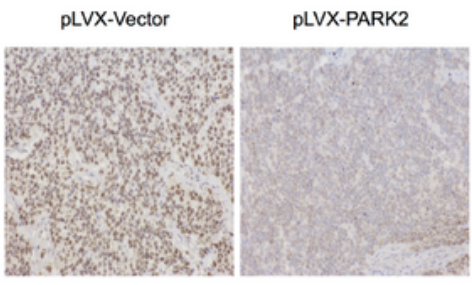

Ki67
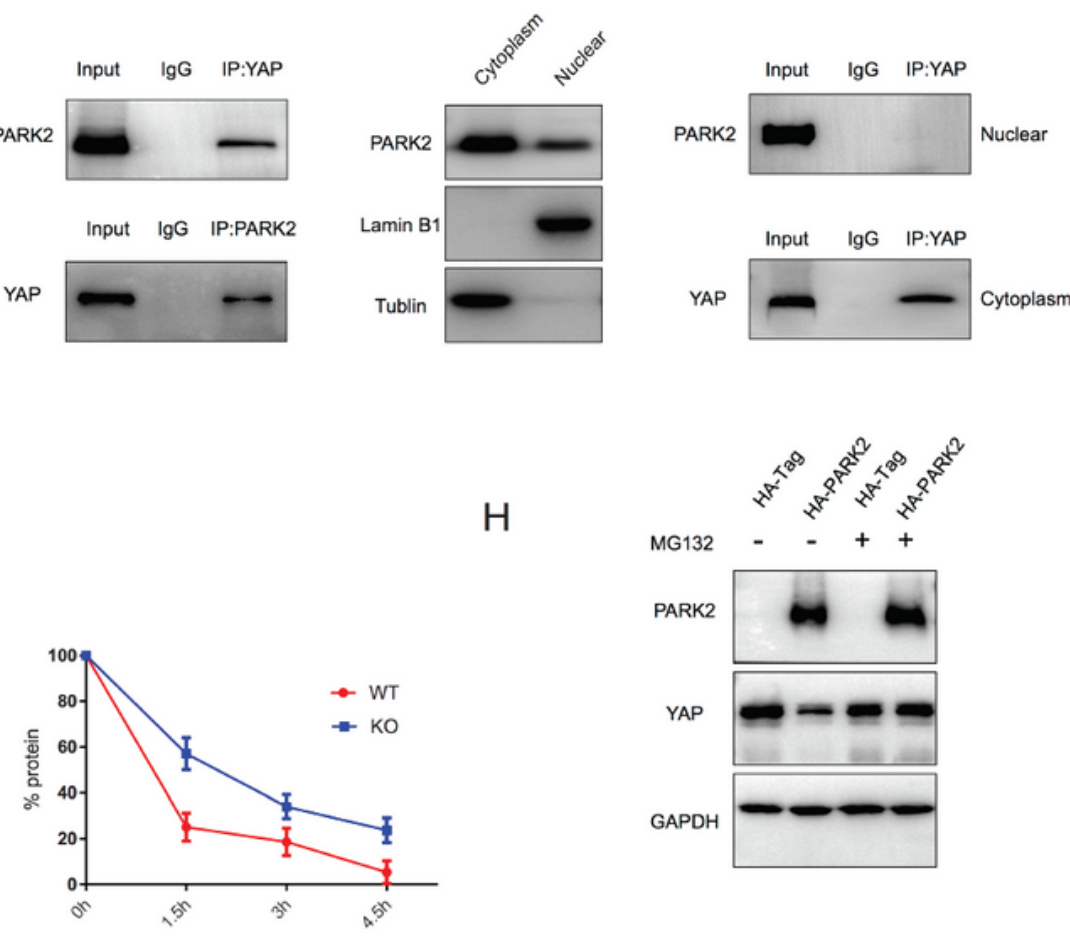

$\mathrm{H}$

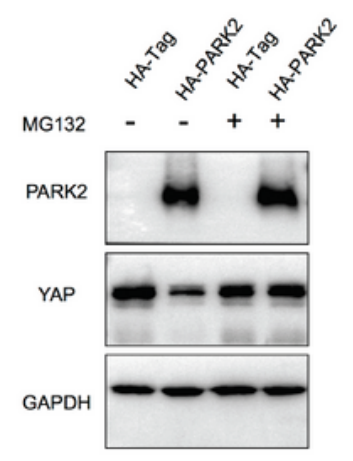

\section{Figure 3}

PARK2 expression is negatively correlated with YAP and interacts with YAP In ESCC cells. A and B. Low PARK2 expression was significantly correlated with increased YAP levels in ESCC specimens analyzed by IHC staining. Scale bar, $100 \mu \mathrm{m}$. C. The negative correlation between YAP and PARK2 expression in xenograft tumors, which is analyzed by IHC staining. Scale bar, $100 \mu \mathrm{m}$. D. IF showed that co-localization of PARK2 (red) and YAP (green). Nuclei were stained with DAPI (blue). Scale bar, 20 um. E. PARK2 
interacted with YAP in ESCC cells. F. PARK2 is mainly localized in the cytoplasm. The subcellular protein fractionation kit was used for cytoplasm and nuclear separation. Tubulin and Lambin B1 were used for cytoplasm and nuclear control. PARK2 interacted with YAP in cytoplasm. G. PARK2 bound to YAP at its Ring domain. (Top panel) Schematic representation of vectors expressing Flag-tagged wild-type or serial deletion mutants of PARK2. (Bottom panel) The Ring domain of PARK2 interacted with YAP. H. PARK2 bound to YAP at its Ring domain. (Top panel) Schematic representation of vectors expressing Flagtagged wild-type or serial deletion mutants of PARK2. (Bottom panel) The Ring domain of PARK2 interacted with YAP. 
A

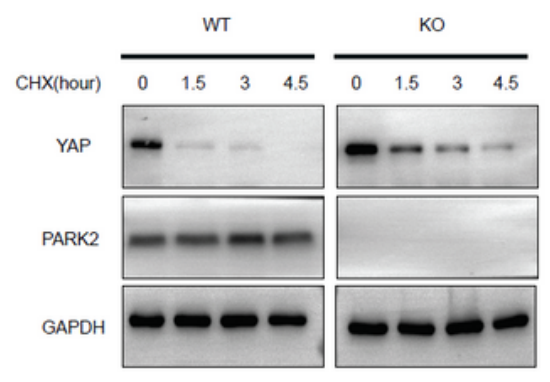

C

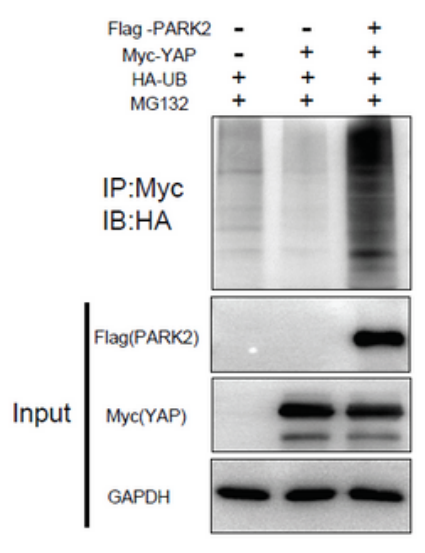

F

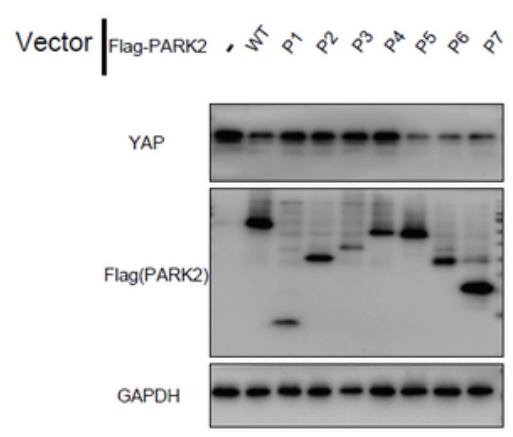

B
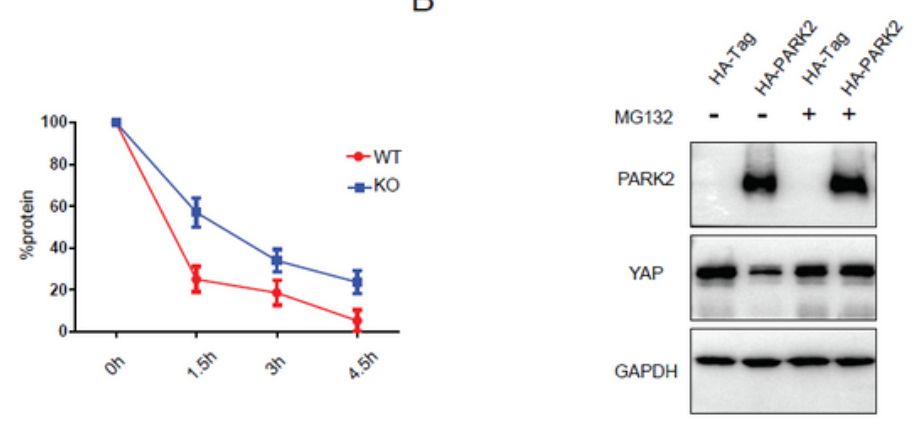

E
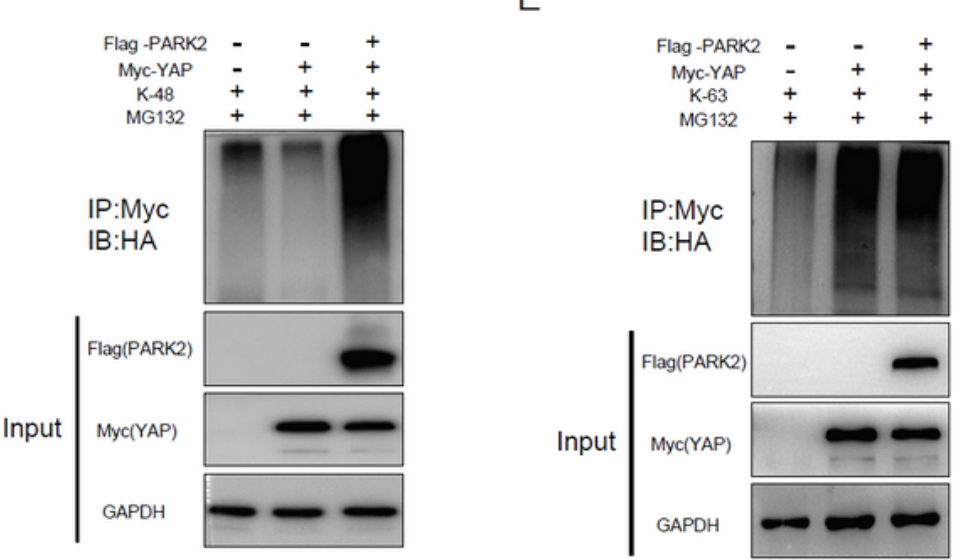

G
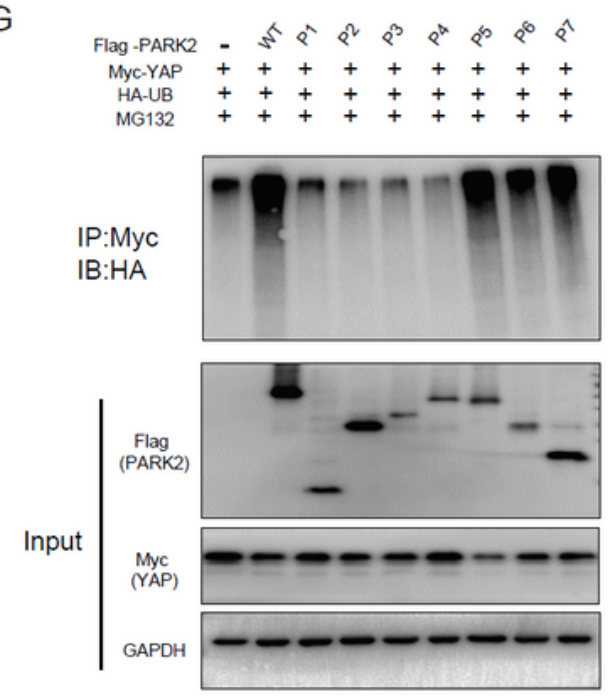

\section{Figure 4}

PARK2 promotes YAP protein degradation through ubiquitination. A. PARK2 knockout decreased YAP protein half-life in EC9706 cells. The cells were treated with $100 \mu \mathrm{mol} / \mathrm{L} \mathrm{CHX}$ for indicated time periods before being collected for western-blot assays. B. PARK2 over-expression could inhibit YAP protein level, which effect could be diminished by MG132. C. Ubiquitin-based Immuno-precipitation showed that PARK2 promoted YAP overall poly-ubiquitination in HEK293T cells. D. Ubiquitin-based Immuno- 
precipitation showed that PARK2 promoted YAP K48-linked ubiquitinaiton in HEK293T cells. E. Ubiquitinbased Immuno-precipitation showed that PARK2 did not affect YAP K63-linked ubiquitinaiton in HEK293T cells. F. The RING domain of PARK2 is required for PARK2 to YAP protein suppression. G. The RING domain of PARK2 is required for PARK2 to regulate ubiquitination of YAP.

A

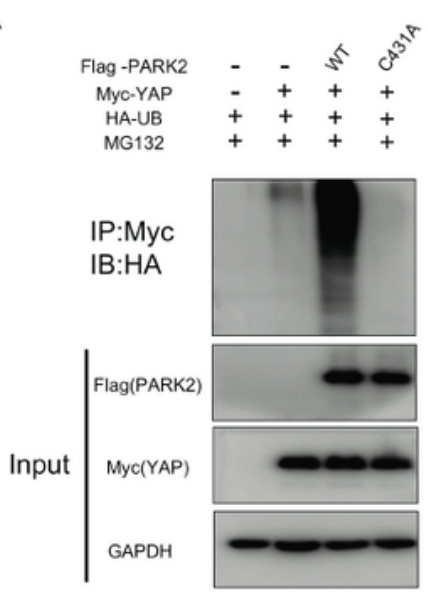

B

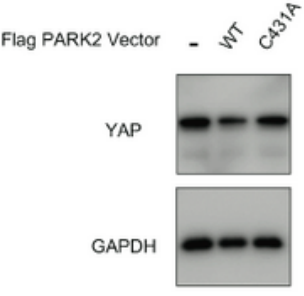

C

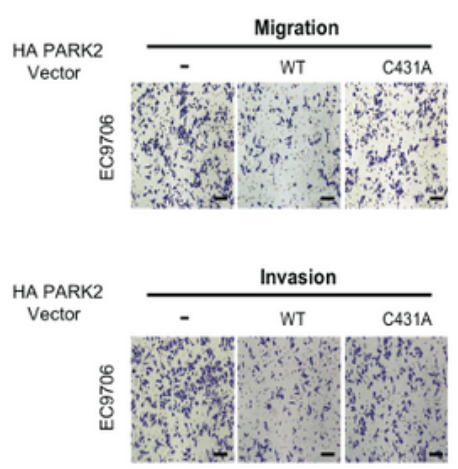

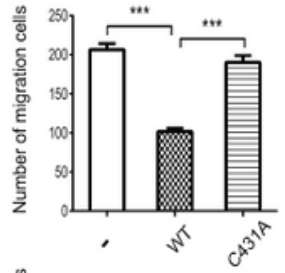

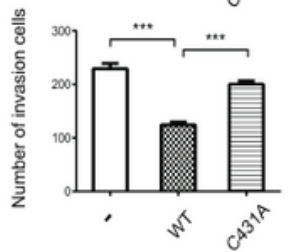

D HA PARK2 Vector

WT

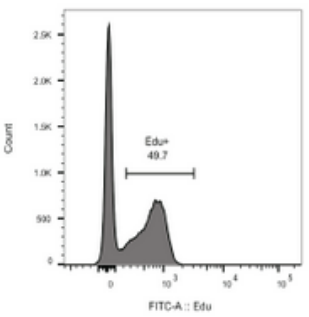

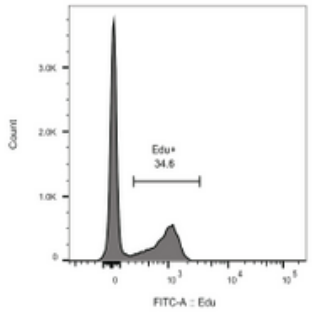

C431A

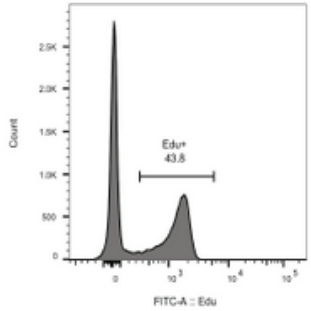

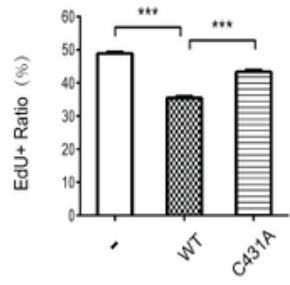

E
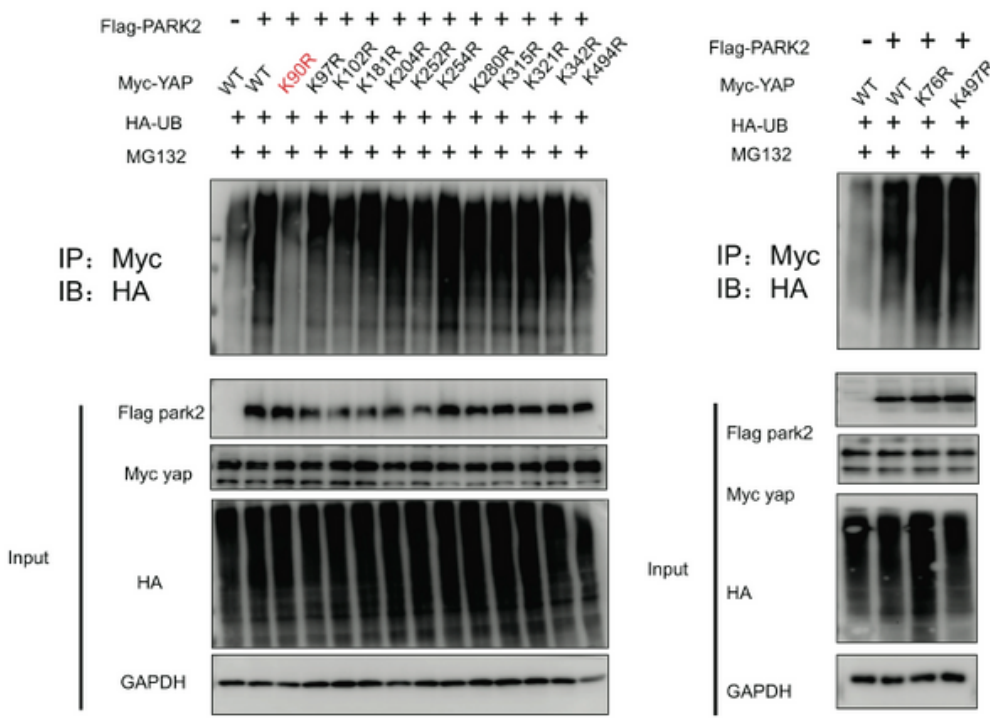

Figure 5 
PARK2 promotes YAP poly-ubiquitination at $\mathrm{K} 90$ site and depends on its ubiquitin ligase activity. $\mathrm{A}$. The effects of expression of Flag PARK2 and its mutants on ubiquitination of Myc YAP in 293T cells analyzed by in vivo ubiquitination assays. B. Mutations of PARK2 that impaired PARK2's ubiquitination activity impaired the ability of PARK2 to degrade YAP protein in EC9706 cells. C. Mutations of PARK2 that impaired PARK2's ubiquitination activity rescued the migration and invasion ability of EC9706 cells with the overexpression of wild type PARK2. Scale bar,100 $\mu$ m. D. Mutations of PARK2 that impaired PARK2's ubiquitination activity rescued the proliferation ability of EC9706 cells with the overexpression of wild type PARK2. E. K90 mutation (K90R) largely abolished ubiquitination of YAP by PARK2. 293T cells were transfected with indicated vectors for in vivo ubiquitination assays.

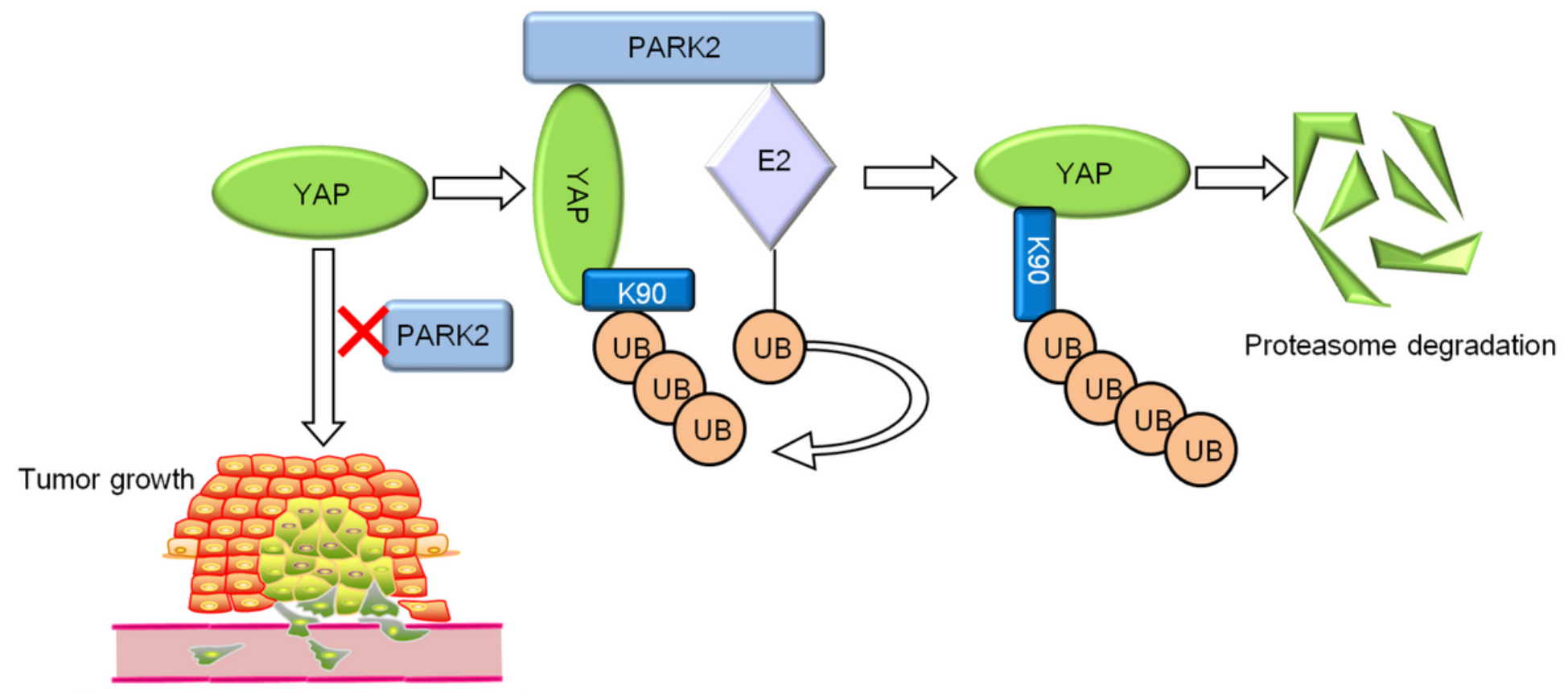

Tumor migration/invasion metastasis

\section{Figure 6}

PARK2 associates with YAP, promotes YAP K48-linked ubiquitination and degradation in ESCC cells, which inhibits the activation of Hippo/YAP axis and ESCC cancer progression.

\section{Supplementary Files}

This is a list of supplementary files associated with this preprint. Click to download.

- supplementarytablePARK2.pdf

- Table1320191221.pdf

- supplementaryfigure.pdf 